\title{
Crack Detection in Single-Crystalline Silicon Wafer Using Laser Generated Lamb Wave
}

\author{
Min-Kyoo Song ${ }^{1}$ and Kyung-Young Jhang ${ }^{2}$ \\ ${ }^{1}$ Graduate School of Mechanical Engineering, Hanyang University, Seoul 133-791, Republic of Korea \\ ${ }^{2}$ School of Mechanical Engineering, Hanyang University, Seoul 133-791, Republic of Korea
}

Correspondence should be addressed to Kyung-Young Jhang; kyjhang@hanyang.ac.kr

Received 19 July 2013; Revised 16 November 2013; Accepted 18 November 2013

Academic Editor: Yan Yang

Copyright ( 2013 M.-K. Song and K.-Y. Jhang. This is an open access article distributed under the Creative Commons Attribution License, which permits unrestricted use, distribution, and reproduction in any medium, provided the original work is properly cited.

\begin{abstract}
In the semiconductor industry, with increasing requirements for high performance, high capacity, high reliability, and compact components, the crack has been one of the most critical issues in accordance with the growing requirement of the wafer-thinning in recent years. Previous researchers presented the crack detection on the silicon wafers with the air-coupled ultrasonic method successfully. However, the high impedance mismatching will be the problem in the industrial field. In this paper, in order to detect the crack, we propose a laser generated Lamb wave method which is not only noncontact, but also reliable for the measurement. The laser-ultrasonic generator and the laser-interferometer are used as a transmitter and a receiver, respectively. We firstly verified the identification of S0 and A0 lamb wave modes and then conducted the crack detection under the thermoelastic regime. The experimental results showed that S0 and A0 modes of lamb wave were clearly generated and detected, and in the case of the crack detection, the estimated crack size by $6 \mathrm{~dB}$ drop method was almost equal to the actual crack size. So, the proposed method is expected to make it possible to detect the crack in the silicon wafer in the industrial fields.
\end{abstract}

\section{Introduction}

Semiconductors have been applied to many fields including smart TVs, smart phones, smart PCs, automobiles, home appliances, and laptop computers. These fields have demanded that the semiconductor should be of high performance, high capacity, high reliability, small components, and low cost. Because of these demands, semiconductor fabrication processes have focused on the lithography for a nanometer-scaled line width. And especially, semiconductor packaging processes have focused on a few trends in recent years: multistacking components and thinner wafers [13]. The multistacking component implies that more chips have to be stacked up within the same size component for the high capacity, and the thinner wafer indicates that wafer thickness has to be very thin up to $30 \mu \mathrm{m}$ for the multistacking component. Moreover, an attempt to change from 12 inch-wafers to 18 inch-wafers is recently going on, since larger wafers which are able to put more chips have impact on the yield improvement. Because of these trends that wafers are getting thinner and larger, silicon wafers are vulnerable to cracks during manufacturing. The cracks can also lead to the yield drop and reliability failure. Therefore, one of the most critical issues in the semiconductor packaging process is the crack. So, the online crack detection in silicon wafers is becoming more important than ever before and its importance cannot be stressed enough.

Previous articles about the online crack detection which deals with poly-crystalline silicon wafers commonly used in the solar cell industry are largely classified into two groups. One is the contact method by resonance ultrasonic vibrations and impact tests $[4,5]$. The other is the noncontact method by an air-coupled ultrasonic lamb wave [6]. Resonance ultrasonic vibrations method can accurately detect the millimeterlength cracks and the impact testing method can also analyze defective wafers. But both of them are driven by external forces which are not adequate for thin silicon wafers under $100 \mu \mathrm{m}$ thickness used generally. Air-coupled ultrasonic lamb wave method demonstrates the crack detection successfully and lamb wave is suitable for the thin silicon wafer. However, 
this method has the problem of high impedance mismatches which cause dissipation of most incident energies from the surface of materials. This is also not satisfied for very noisy environment caused from facilities or manufacturing machines.

For many years, laser-ultrasonic technology has been increasingly used for nondestructive crack detections [710]. The biggest advantage of this technology is noncontact without regard to external forces and surface conditions of materials. This technology has another advantage that can reduce the energy loss by adjusting the shape of the laser beam for the energy enhancement. Although laserultrasonic technology has these advantages, few studies have performed for the crack detection in silicon wafers. Therefore, we propose the laser generated lamb wave method, which not only is noncontact but also guarantees the reliable measurement. And this paper deals with single crystalline silicon wafers commonly used in the semiconductor industry other than previous papers which deal with poly-crystalline silicon wafers.

Laser-ultrasonic technology has two main regimes. One is thermoelastic regime and the other is ablation regime [11]. In the case of thermoelastic regime, the ultrasonic waves are generated from thermoelastic expansions of materials. Meanwhile, in the case of ablation regime, the ultrasonic waves are generated from the material removal. If the ablation regime is applied to silicon wafers, they will be damaged. So, thermoelastic regime has to be applied to silicon wafers in terms of the reliability. And also there are two possible generation mechanisms; thermal effect and electrostriction. However, the thermal effect is dominant at the high power input while the electrostriction is dominant at the low power input [12]. In our case, the input power was in the range of high power input, so that we did not consider the electrostriction effect.

If the thermoelastic regime is applied to a silicon wafer that has the comparable thickness to wavelength, lamb wave is generated. We are expected to detect the cracks in silicon wafers through analysis of this lamb wave.

\section{Dispersion Curves of Lamb Wave in Silicon Wafer}

Lamb wave is dispersive, which implies that the wave velocity is dependent on the frequency. So dispersion curves need to be analyzed first. The wave velocities in single-crystalline silicon wafers are listed in Table 1 . The silicon wafer has three kinds of different velocities depending on the orientations [13]. But, since wafers have the characteristics that cracks are easily initiated and cleft in the (110) orientation, it is reasonable to consider only the (110) orientation in terms of easier experiments. Figure 1 shows dispersion curves of group velocity which are plotted from velocities at the (110) orientation in the silicon wafer whose thickness is $525 \mu \mathrm{m}$. The curves in the dotted box area are mostly used for the practical applications, because other areas are more complicated. So the curves in this box area are supposed to be used for our experiments as well.
TABLE 1: Wave velocities in single-crystalline silicon wafer.

\begin{tabular}{lcc}
\hline Orientation & Longitudinal wave velocity & Shear wave velocity \\
\hline$(100)$ & $8436(\mathrm{~m} / \mathrm{s})$ & $5847(\mathrm{~m} / \mathrm{s})$ \\
$(110)$ & $9138(\mathrm{~m} / \mathrm{s})$ & $4675(\mathrm{~m} / \mathrm{s})$ \\
$(111)$ & $9360(\mathrm{~m} / \mathrm{s})$ & $5096(\mathrm{~m} / \mathrm{s})$ \\
\hline
\end{tabular}

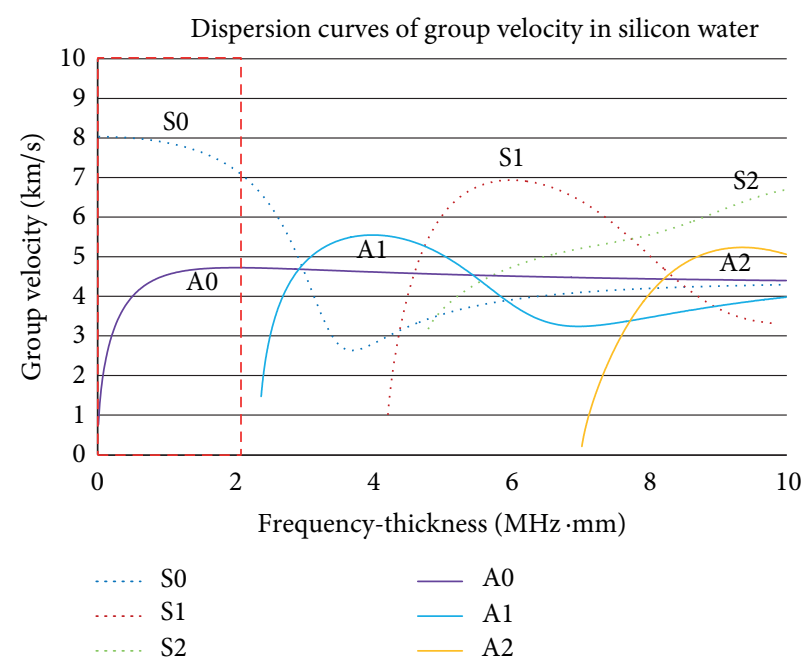

FIGURE 1: Dispersion curves of group velocity in silicon wafers whose thickness is $525 \mu \mathrm{m}$.

\section{Simulation}

The thermoelastic mechanism is described by a few equations. First, the laser energy that has Gaussian spatial and temporal distributions is quickly absorbed in the wafer as shown in Figure 2. This process can be expressed as

$$
Q=2 \frac{E(1-R)}{S \tau} \exp \left(-2 \frac{x^{2}}{r^{2}}\right) \exp \left(2.7726 \frac{\left(t-2 \tau^{2}\right)}{\tau^{2}}\right)
$$

where $Q$ means the heat source; $E$ is the initial laser energy; $R$ is the reflectivity; $S$ is the surface area on which the laser is absorbed; $\tau$ is the pulse duration; $r$ indicates a distance that laser density is $1 / 2$ times from the center; $x$ is the spatial coordinates; $t$ means time.

And then, the absorbed energy makes the temperature of the wafer increase sharply. The thermal conduction is caused by this large temperature gradient. This thermal conduction equation can be described as

$$
\rho C_{p} \frac{\partial T}{\partial t}+\rho C_{p} u \nabla T=\nabla(k \nabla T)+Q
$$

where $\rho, C_{p}$, and $T$ are density, heat capacity, and temperature, respectively; $u$ means the displacement; $Q$ is the heat generated from the heat source. 
TABLE 2: Properties of the wafer.

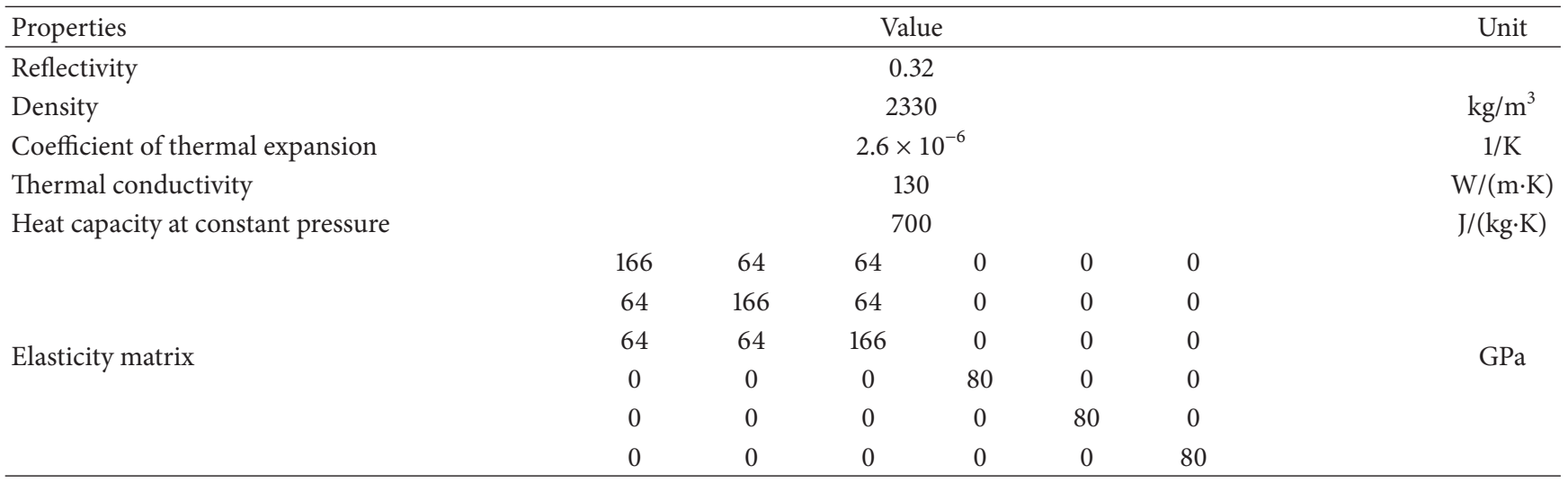

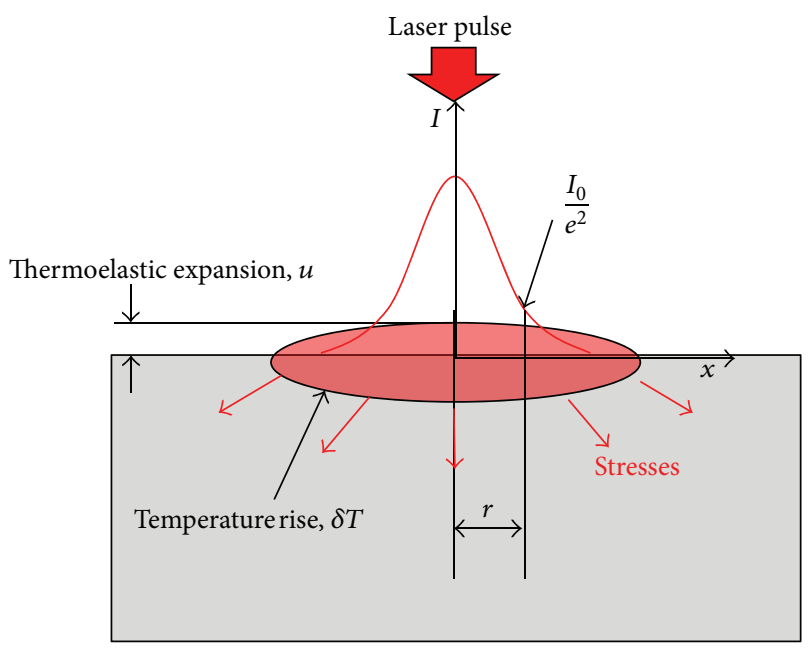

Figure 2: Schematic of thermoelastic mechanism.

At last, thermoelastic expansion is caused by the large temperature gradient. Thermoelastic stresses are finally generated and thermoelastic wave is rapidly propagated. This equation of motion can be represented as

$$
(\lambda+2 \mu) \nabla(\nabla \cdot u)-\mu \nabla \times \nabla \times u-\alpha(3 \lambda+2 \mu) \nabla T=\rho \frac{\partial^{2} u}{\partial t^{2}},
$$

where $\lambda$ and $\mu$ are Lamé constants; $\alpha$ is coefficient of thermal expansion.

All of these equations were applied to the simulation with COMSOL multiphysics 4.3. The incident laser density and the pulse duration were adopted as $1.00 \times 10^{12} \mathrm{~W} / \mathrm{m}^{2}$ and $5 \mathrm{~ns}$. And we considered the line beam as the elliptical beam which has $11.5 \mathrm{~mm}$ major axis and $0.5 \mathrm{~mm}$ minor axis, as shown in the next experiment section. Other properties used in the simulation are listed in Table 2 . The geometrical dimensions of this simulation were modeled as shown in Figure 3: the diameter of the wafer, 4 inches and the thickness of the wafer, $525 \mu \mathrm{m}$. The outside boundaries were considered as thermal insulations. And the mesh size was determined as $0.1 \mathrm{~mm}$

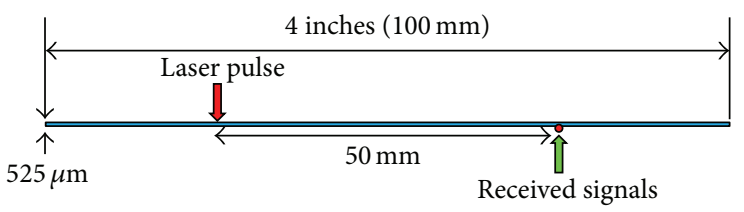

FIGURE 3: Geometrical dimensions of simulation model.

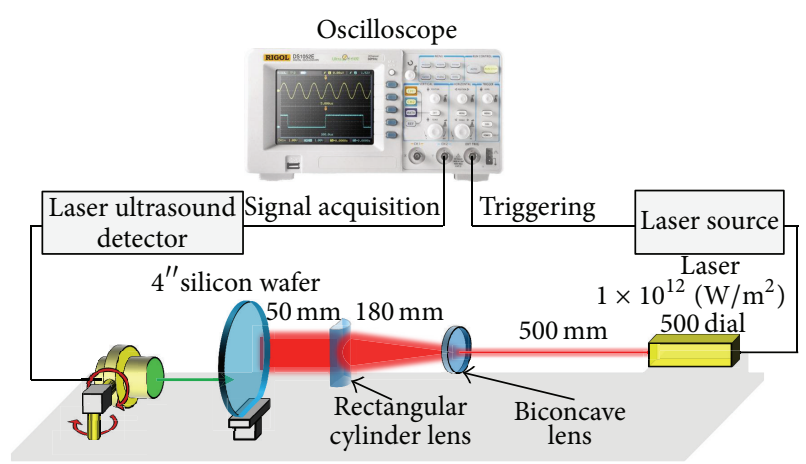

FIgURE 4: Experimental setup.

which is less than a 10th of the A0 mode wavelength and is small enough to get reasonable calculation accuracy [14]. Lamb wave was obtained at a distance of $50 \mathrm{~mm}$ from the laser source in this simulation.

\section{Experimental Procedures and Setup}

We conducted two experiments that are mode identification and crack detection. Firstly, lamb wave modes were identified in the wafer without any crack, as comparing the experiment and the simulation. Secondly, the modes which were identified in the first experiment were used for the crack detection experiment in the wafer with an artificial crack.

Figure 4 illustrates the experimental setup. The materials we used for two experiments are (100) single-crystalline silicon wafers that are manufactured by ShinEtsu Chemical 


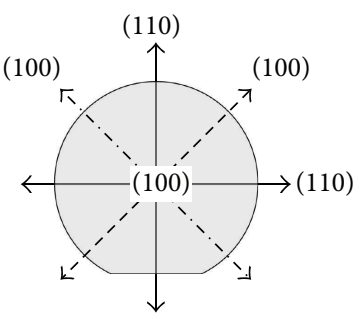

(a)

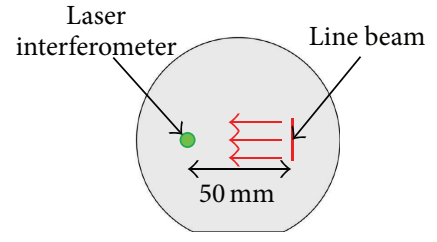

(b)
Figure 5: (a) Orientation in a (100) wafer and (b) the setup of the laser source and the laser interferometer.

Co., Ltd. The diameter and thickness of the wafers are 4 inches and $525 \mu \mathrm{m}$, respectively, which are the same in the previous simulation section. The pulsed laser made by Spectron Laser Systems was used as a transmitter. Its fundamental wavelength, beam diameter, pulse duration, and the repetition rate are $1064 \mathrm{~nm}, 4 \mathrm{~mm}, 5 \mathrm{~ns}$, and $1 \mathrm{~Hz}$, respectively. The laser interferometer made by TECNAR was used as a receiver. Its operating principle is described by two-wave mixing. This equipment detects out-of-plane displacement and the frequency bandwidth for the detection of lamb wave has upper limit, $2 \mathrm{MHz}$. An oscilloscope was used for triggering and signal acquisition.

Two lenses, which are able to transform the laser beam into a line beam, were used to make it clear to generate lamb wave: a biconcave lens and a rectangular cylinder lens. The biconcave lens was placed at a distance of $500 \mathrm{~mm}$ from the laser and then the distance between the biconcave lens and the rectangular cylinder lens was set to $180 \mathrm{~mm}$, in order to make a beam diameter of $23 \mathrm{~mm}$. The silicon wafer was located where the laser beam became a line beam whose width was $1 \mathrm{~mm}$ and length was $23 \mathrm{~mm}$. Its distance was $50 \mathrm{~mm}$ from the rectangular cylinder lens. The laser intensity of the line beam was $1.00 \times 10^{12} \mathrm{~W} / \mathrm{m}^{2}$, which is within the thermoelastic regime and less than the ablation threshold. The laser interferometer was installed behind the wafer due to the interference with other equipment. The oscilloscope was linked both to the laser source for the triggering and to the laser interferometer for the signal acquisition.

\section{Mode Identification}

The experimental setup for the mode identification was the same as in Figure 4. In addition, the orientation of the wafer was taken into account, because we supposed to only consider the (110) orientation where cracks are easily generated. Figure 5(a) illustrates the orientations in a (100) wafer. The line beam as a laser source was aligned perpendicular to the flat zone of the wafer, in order to generate lamb wave in the direction, (110), as shown in Figure 5(b) which describes the setup of the laser source and the laser interferometer.

In the case of the experimental result, the triggering signal is occupied by around $4 \mu \mathrm{s}$ as shown in Figure 6(a). We can also see that S0 mode and A0 mode are clearly generated and received. S0 mode is received in $6.24 \mu \mathrm{s}$ and A0 mode is measured from $8.5 \mu$ s to $18 \mu \mathrm{s}$.
In the case of the simulation result, S0 mode and A0 mode are also obviously generated and received. S0 mode is obtained in $6.6 \mu$ s and A0 mode is followed for the time which is similar to the experimental result, as shown in Figure 6(b).

As comparing the experiment with the simulation, the arrival times of S0 mode are almost the same. Likewise, the arrival times of A0 mode are also the same. In terms of the velocity, S0 mode is faster than A0 mode. In terms of the amplitude, however, it can be seen from both results that the amplitude of A0 mode is much larger than that of S0 mode. This is because A0 mode is dominant for out-of-plane on the surface of the wafer; on the other hand, S0 mode is dominant for in-plane on the surface of the wafer. Note that the equipment used for the receiver detects only the out-ofplane displacement. As a result of plotting the STFT result overlapped with the group velocity, A0 mode strongly appears around $500 \mathrm{kHz}$ as shown in Figure 7. It implies that A0 mode is helpful for the crack detection.

However, considering that the nondispersive wave is preferable for the easier interpretation of wave signal, the higher frequency band of $2 \sim 4 \mathrm{MHz}$ looks better as we can see from the dispersion curve of A0 mode shown in Figure 7. But, in our experimental setup, the upper frequency limit of lamb wave detector was $2 \mathrm{MHz}$ and the line beam width $1 \mathrm{~mm}$ was the least width for the safe generation in the thermoelastic regime. Thus, we did not have much flexibility to choose the frequency band.

In order to use the higher frequency lamb wave for the inspection, both sides of generation and detection should be considered. In the generation side, a simple way is the reduction of the line beam width. But, in this case, there exists a limit in the input laser power to keep the laser intensity of line beam within the thermoelastic range. In the detection side, the frequency bandwidth and the sensitivity should be considered. The frequency bandwidth of lamb wave detector is dependent on the beam spot size of detection laser. The smaller spot size can detect the higher frequency (or the shorter wavelength) lamb wave. Also, if we reduce the generation laser power to make the line beam width smaller within the thermoelastic regime, then the generated wave amplitude will be decreased. In this case, the higher sensitivity of detector may be required.

\section{Crack Detection}

A0 mode was used for the crack detection because of the large amplitude, as was mentioned in the former section. The experimental setup was nothing different from the setup for the mode identification. But, it is the difference that the reflection method was used by scanning the wafer that has the crack artificially made with a diamond cutter, as shown in Figure 8. The crack length, width, and depth are $5 \mathrm{~mm}$, $0.5 \mathrm{~mm}$, and $0.1 \mathrm{~mm}$, respectively.

We analyzed what kinds of modes are generated in the center of the crack prior to the crack detection experiment. The crack, the laser interferometer, and the line beam were positioned as shown in Figure 9(a). And then a variety of modes were received as shown in Figure 9(b). S0 mode arrives the earliest at $2.5 \mu \mathrm{s}$, but this is buried in the triggering 


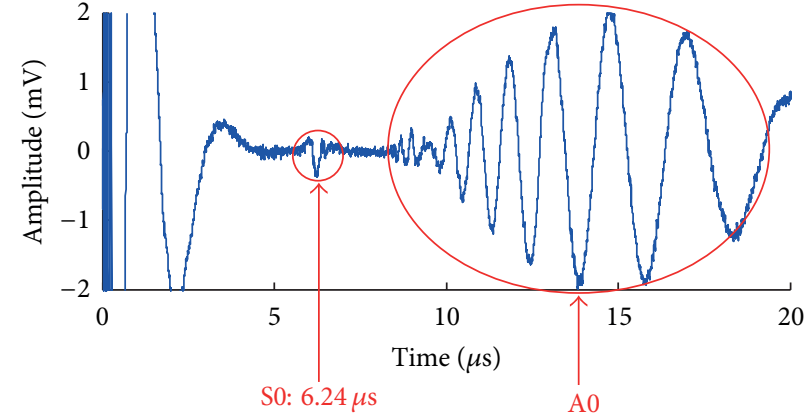

(a)

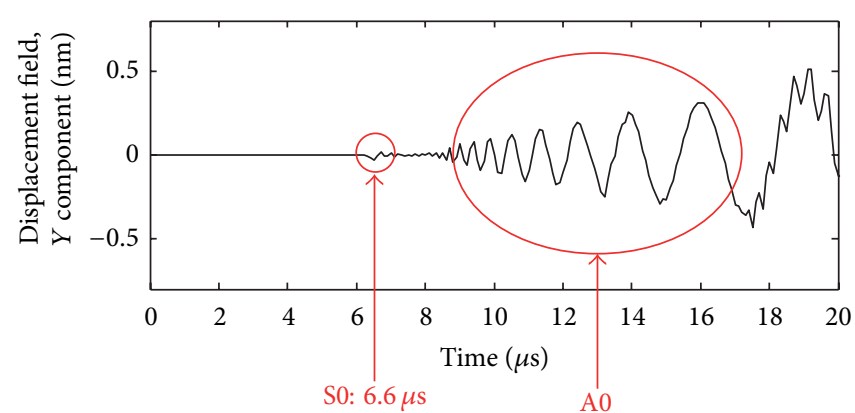

(b)

FIGURE 6: (a) Experimental result and (b) simulation result for mode identification.

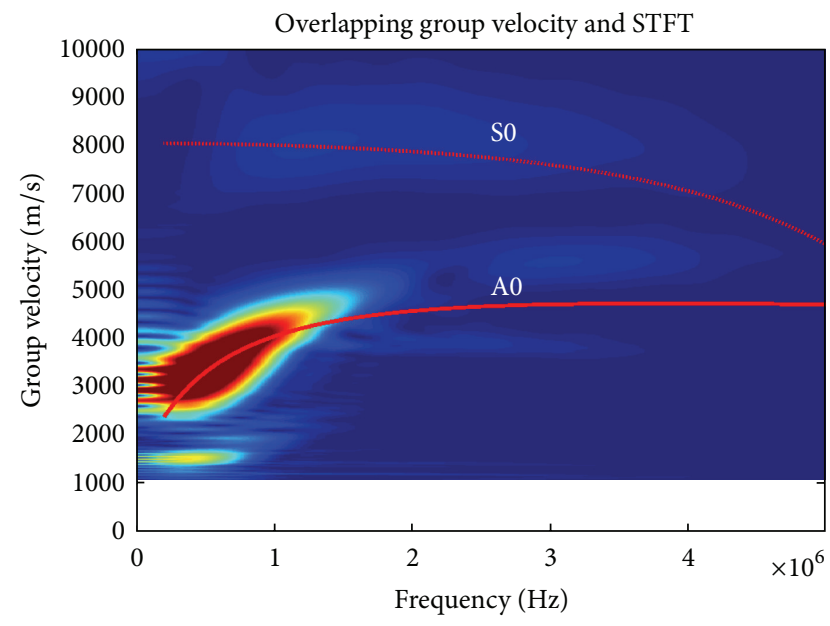

FIGURE 7: STFT result overlapped with group velocity.

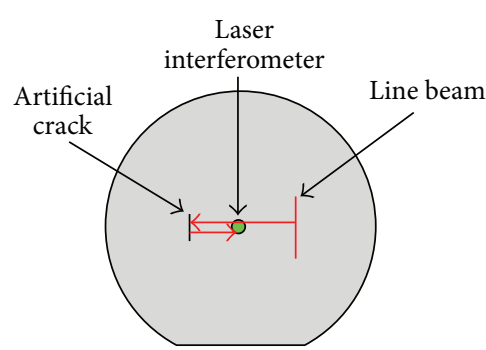

(a)

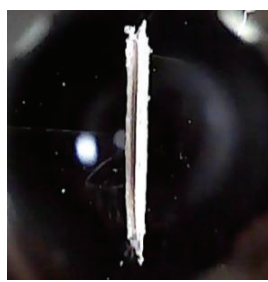

(b)
FIGURE 8: (a) Reflection method schematic and (b) artificial crack.

signal. Since S0 mode has very tiny amplitude, it will not be considered any more. And A0 mode arrives then at $6.07 \mu \mathrm{s}$. Some part of A0 mode is also buried in the triggering signal. Next, A0 mode reflected from the crack is detected at $15.17 \mu \mathrm{s}$. This is what we are looking for in this experiment. And then, the complicated signals are followed. So the reflected A0 mode from the crack is analyzed for the crack detection and its signal is in the range from 12 to $18 \mu \mathrm{s}$.

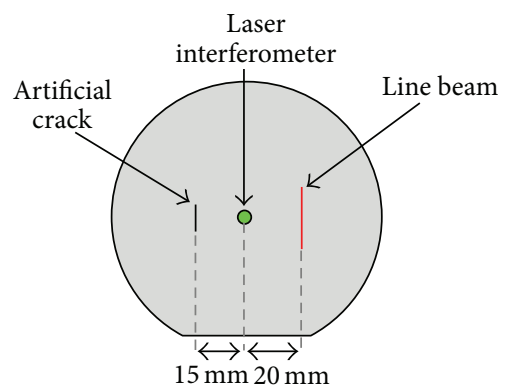

(a)

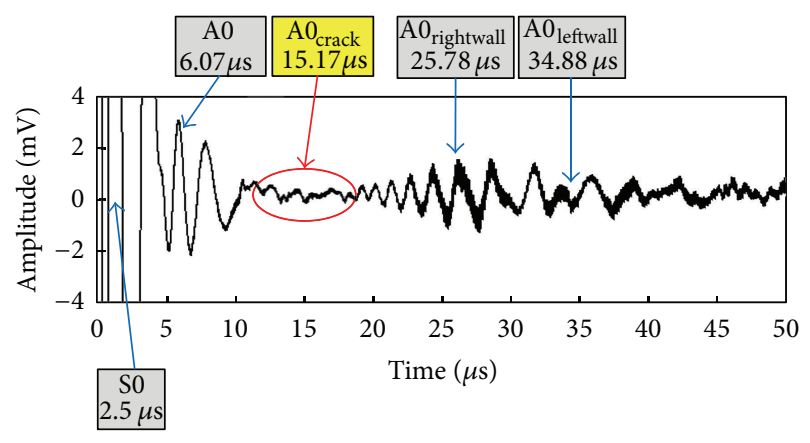

(b)

Figure 9: (a) Experimental setup and (b) received signals.

The crack was scanned with every $0.5 \mathrm{~mm}$ and the scan length was $10 \mathrm{~mm}$ as shown in Figure 10(a). So every datum corresponding to each A0 mode reflected from the crack was extracted and plotted based on the maximum amplitude in the range from 12 to $18 \mu \mathrm{s}$. And then the $6 \mathrm{~dB}$ drop method is applied to decide the defect size, which has been widely used for the defect sizing based on the ultrasonic scan $[15,16]$. Figure 10(b) shows the experimental result that the estimated crack length by $6 \mathrm{~dB}$ drop method is about $4.8 \mathrm{~mm}$. In comparison with the actual crack length, $5 \mathrm{~mm}$, we come to conclusion that the width more than $50 \%$ of the maximum signal level can be considered as an actual crack length. Therefore, the proposed method is expected to make it possible for the online crack detection in the silicon wafer. 


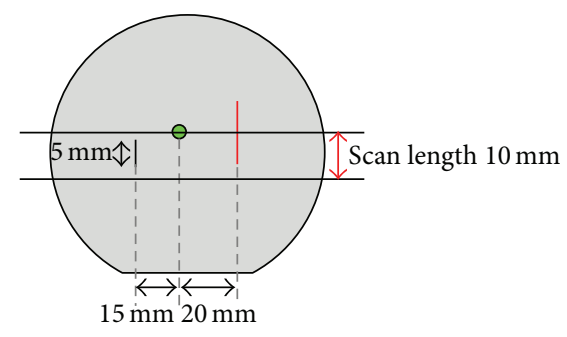

(a)

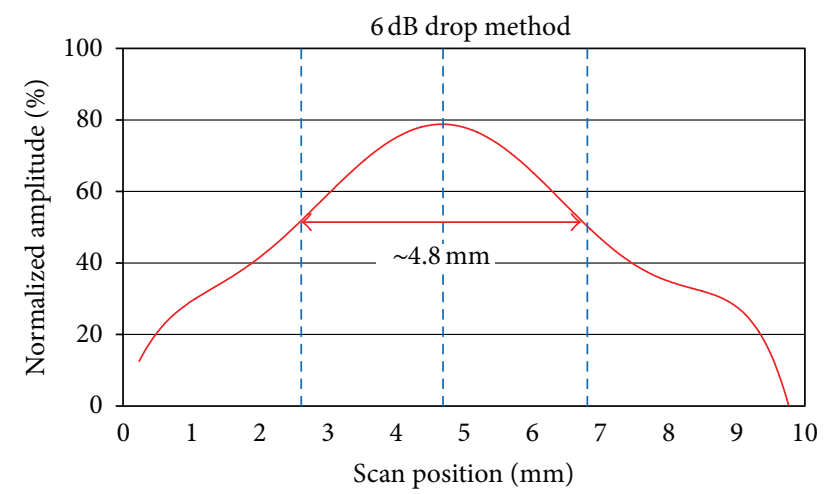

(b)

Figure 10: (a) Scan length and (b) experimental result by $6 \mathrm{~dB}$ drop method.

\section{Conclusions}

This paper proposed the laser generated Lamb wave which not only is noncontact method, but also guarantees the reliable measurement, in order to detect the crack in the single-crystalline silicon wafer. We verified the identification of S0 and A0 lamb wave modes and then conducted crack detection under the thermoelastic regime. At first, mode identification was carried out, as comparing the experiment with the simulation. The experimental result showed a good agreement with the simulation result. In particular, we could see that the amplitude of A0 mode is remarkably larger than that of S0 mode. This is because A0 mode is dominant for out-of-plane; on the other hand, S0 mode is dominant for in-plane. So A0 mode was used for the crack detection. It can be seen that the actual crack size was nearly identical to the estimated crack size by $6 \mathrm{~dB}$ drop method, as the crack was scanned. Finally, we concluded that laser generated lamb wave we proposed can be used for the crack detection in silicon wafer.

Although this paper deals with a single-crystalline silicon wafer as the beginning of the crack detection, our future work will deal with an individual chip separated from a thinner wafer by a saw machine.

\section{Acknowledgment}

This work was financially supported by the National Research Foundation of Korea (NRF) as a grant funded by the Korean government (NRF-2013M2A2A9043241).

\section{References}

[1] E.-K. Kim, "Assessment of ultra-thin Si wafer thickness in 3D wafer stacking," Microelectronics Reliability, vol. 50, no. 2, pp. 195-198, 2010.

[2] C.-C. A. Chen and L.-S. Hsu, "A process model of wafer thinning by diamond grinding," Journal of Materials Processing Technology, vol. 201, no. 1-3, pp. 606-611, 2008.

[3] Y. S. Kim, N. Maeda, H. Kitada et al., "Advanced wafer thinning technology and feasibility test for 3D integration," Microelectronic Engineering, vol. 107, pp. 65-71, 2013.

[4] W. Dallas, O. Polupan, and S. Ostapenko, "Resonance ultrasonic vibrations for crack detection in photovoltaic silicon wafers," Measurement Science and Technology, vol. 18, no. 3, article 038, pp. 852-858, 2007.

[5] C. Hilmersson, D. P. Hess, W. Dallas, and S. Ostapenko, "Crack detection in single-crystalline silicon wafers using impact testing," Applied Acoustics, vol. 69, no. 8, pp. 755-760, 2008.

[6] S. K. Chakrapani, M. J. Padiyar, and K. Balasubramaniam, "Crack detection in full size Cz-silicon wafers using lamb wave air coupled ultrasonic testing (LAC-UT)," Journal of Nondestructive Evaluation, vol. 31, pp. 46-55, 2012.

[7] C. Ni, Y. Shi, Z. Shen, J. Lu, and X. Ni, "An analysis of angled surface-breaking crack detection by dual-laser source generated ultrasound," NDT and E International, vol. 43, no. 6, pp. 470475, 2010.

[8] S. Dixon, S. E. Burrows, B. Dutton, and Y. Fan, "Detection of cracks in metal sheets using pulsed laser generated ultrasound and EMAT detection," Ultrasonics, vol. 51, no. 1, pp. 7-16, 2011.

[9] C. Ni, L. Dong, Z. Shen, and J. Lu, "The experimental study of fatigue crack detection using scanning laser point source technique," Optics and Laser Technology, vol. 43, no. 8, pp. 13911397, 2011.

[10] S. J. Davies, C. Edwards, G. S. Taylor, and S. B. Palmer, "Laser-generated ultrasound: its properties, mechanisms and multifarious applications," Journal of Physics D, vol. 26, no. 3, pp. 329-348, 1993.

[11] C. B. Scruby and L. E. Drain, Laser Ultrasonics: Techniques and Applications, Adam Hilger, Bristol, UK, 1990.

[12] H. J. Shin, S. W. Noh, J. H. Lee, and J. S. Jang, "Pulsed laser induced photoacoustic effect in silicon, mainly caused by thermal expansion and electrostriction, is studied," Journal of the Institute of Electronics Engineers of Korea, vol. 3, pp. 42-44, 1988.

[13] J. J. Wortman and R. A. Evans, "Young's modulus, shear modulus, and poisson's ratio in silicon and germanium," Journal of Applied Physics, vol. 36, no. 1, pp. 153-156, 1965.

[14] H.-X. Sun, S.-Y. Zhang, and B.-Q. Xu, "Influence of viscoelastic property on laser-generated surface acoustic waves in coatingsubstrate systems," Journal of Applied Physics, vol. 109, no. 7, Article ID 073107, 2011.

[15] J. Blitz and G. Simpson, Ultrasonic Methods of Non-Destructive Testing, Chapman and Hall, London, UK, 1996.

[16] P. Ciorau, "Comparison between $-6 \mathrm{~dB}$ and $-12 \mathrm{~dB}$ amplitude drop techniques for length sizing," Ontario Power Generation, no. 11, 2011, http://www.ndt.net/. 

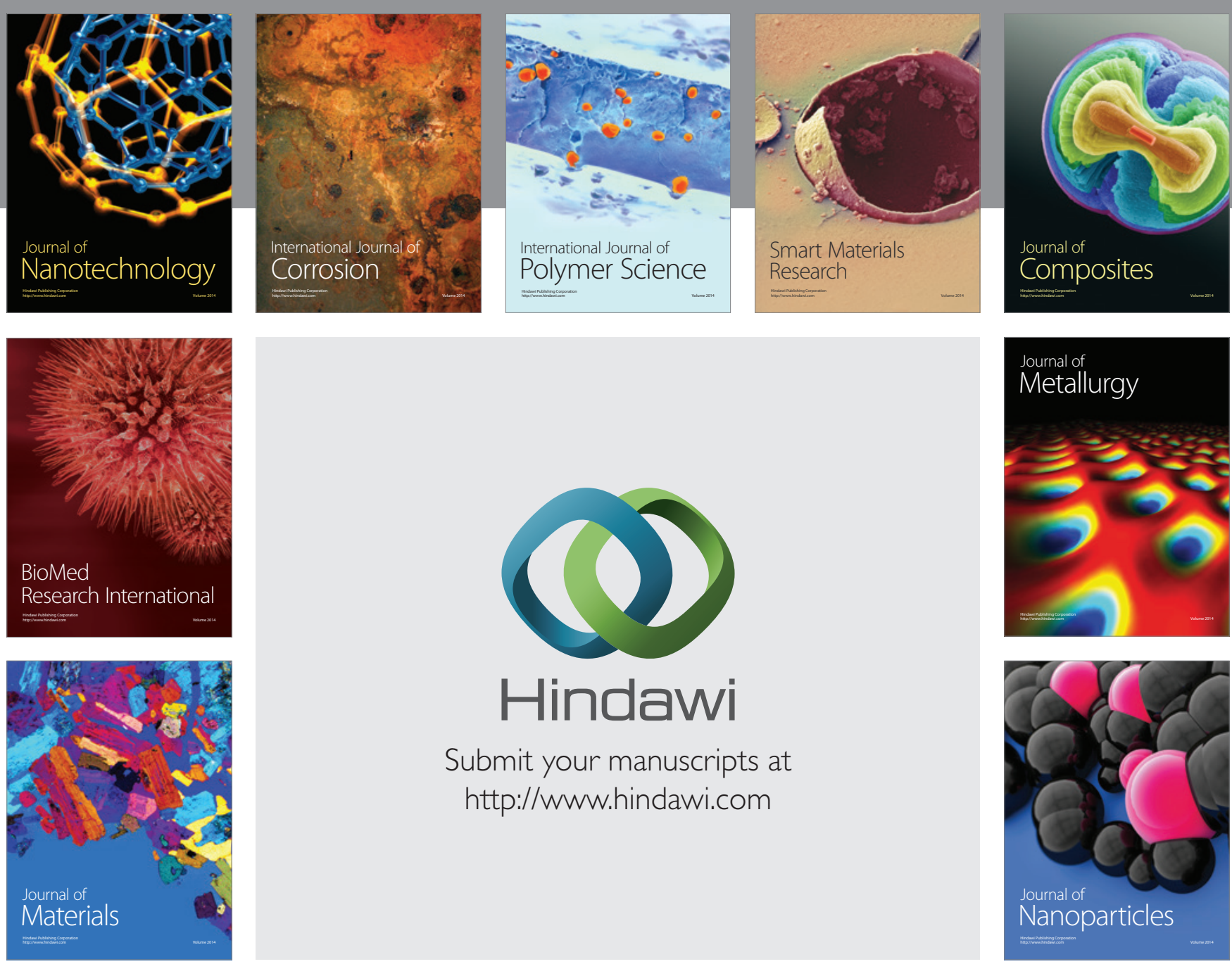

Submit your manuscripts at http://www.hindawi.com
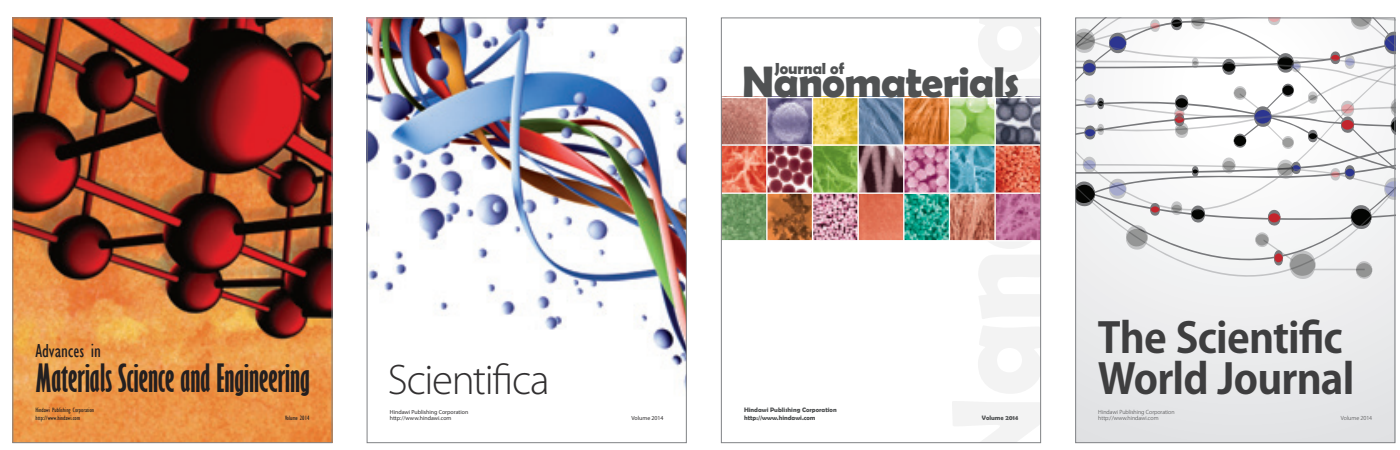

\section{The Scientific World Journal}
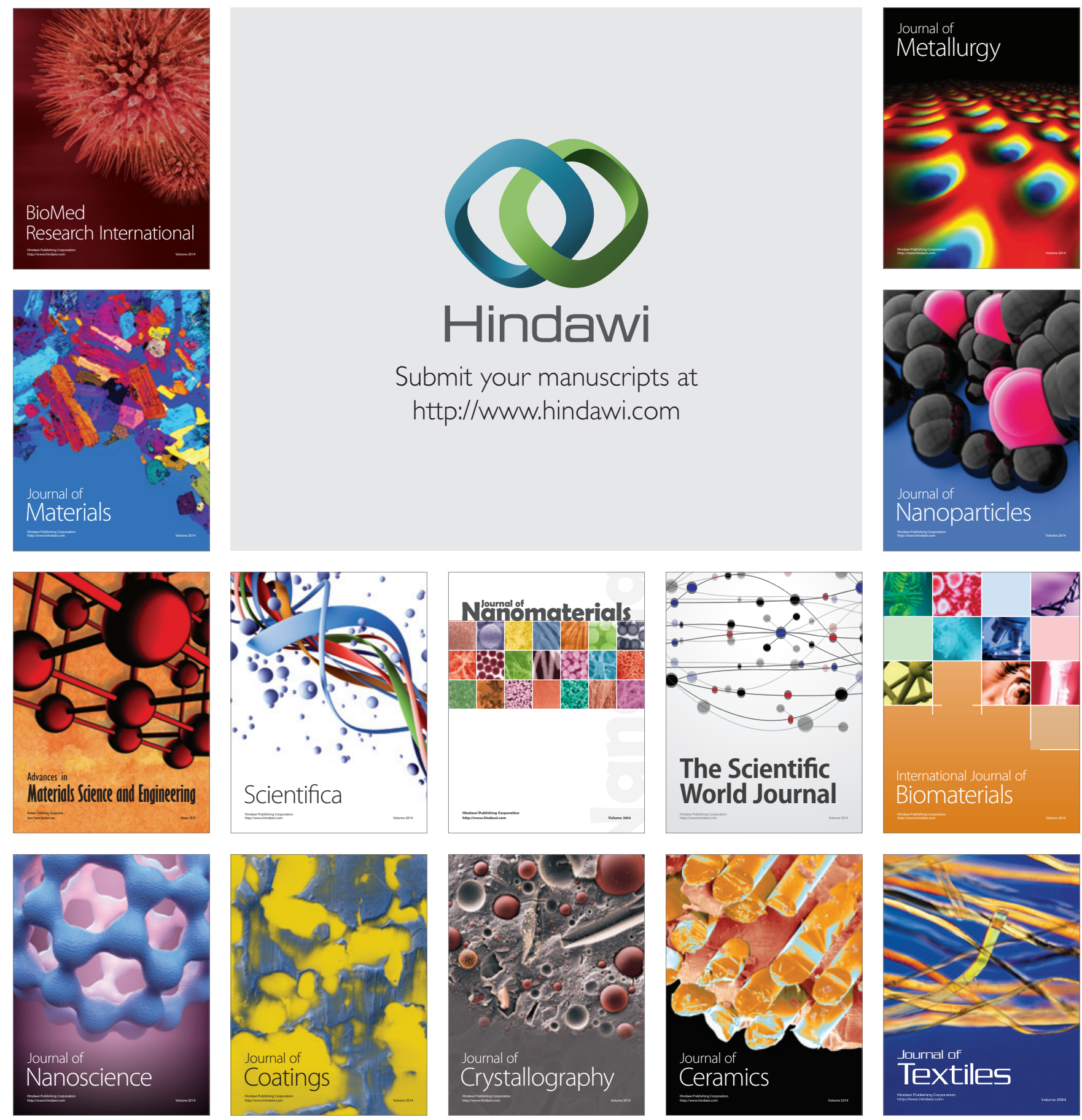